\title{
Does Preeclampsia Predict the Risk of Late Postpartum Eclampsia?
}

\author{
Diana S. Wolfe, MD, MPH ${ }^{1}$, Shauna F. Williams, MD ${ }^{2}$ Michael G. Ross, MD, MPH ${ }^{1}$ Marie H. Beall, MD ${ }^{1}$ \\ Joseph J. Apuzzio, $\mathrm{MD}^{2}$ \\ ${ }^{1}$ Department of Obstetrics and Gynecology, Albert Einstein College of \\ Medicine, Montefiore Medical Center, New York, New York \\ 2 University of Medicine and Dentistry of New Jersey, Newark, New \\ Jersey \\ Address for correspondence Diana S. Wolfe, MD, Department of \\ Obstetrics and Gynecology, Albert Einstein College of Medicine, \\ Montefiore Medical Center, New York, New York, 1825 Eastchester Rd, \\ Room 701, Bronx, NY 10641 (e-mail: dwolfe@montefiore.org).
}

Am J Perinatol Rep 2013;3:13-16.

\begin{abstract}
Objective To investigate potential predictive symptoms of late postpartum eclampsia (LPE).

Study Design Retrospective review of patients delivered at a single academic medical center and diagnosed with eclampsia greater than 48 hours postdelivery.

Results Among 19 patients with eclampsia, 5 (26\%) patients with confirmed eclampsia

\section{Keywords}

- postpartum eclampsia

- atypical eclampsia

- eclampsia

- late postpartum eclampsia (LPE) seized greater than 48 hours after delivery. None of these patients showed evidence of preeclampsia intrapartum or immediately postpartum and none received intrapartum magnesium sulfate. Prior to seizure activity, 4 of 5 (80\%) patients had increased blood pressure and 2 of 5 (40\%) had central nervous system symptoms (headache and visual changes).

Conclusion Gestational hypertension (GHTN) may be a risk factor for LPE. Consideration of seizure prophylaxis for patients with GHTN may facilitate the prevention of LPE.
\end{abstract}

Preeclampsia is diagnosed in pregnant women by the onset of hypertension and proteinuria. In the most severe cases, maternal seizures (eclampsia) may ensue. Both eclampsia and preeclampsia may result in serious morbidity or mortality, with a marked increase in the maternal death rate associated with eclampsia. ${ }^{1}$ It is therefore prudent to treat preeclampsia before it develops into eclampsia, which most commonly occurs proximate to delivery.

Late postpartum eclampsia (LPE) occurring more than 48 hours after delivery has been a relatively uncommon condition. LPE may occur without warning, as the typical clinical features of preeclampsia may not be present until after the onset of seizure activity. The relative incidence of LPE appears to be increasing. Glasgow Royal Maternity Hospital reported a relative increase in the proportion of postpartum eclampsia during the years 1931 to 1990 compared with antenatal and intrapartum eclampsia. ${ }^{2}$ Prediction of patients at risk of LPE is challenging as factors associated with postpartum eclampsia may be different from those related to antepartum and intrapartum disease. Lubarsky et al suggest that one possible explanation for the increased proportion of postpartum eclampsia may be early and aggressive use of magnesium sulfate therapy in the antepartum and immediate postpartum periods, resulting in a shift in the proportion of cases. ${ }^{3}$

We reviewed our experience at the University Hospital of UMDNJ (University of Medicine and Dentistry of Newark, NJ) to determine if there were common clinical factors prior to seizure activity in LPE patients.

\section{Materials and Methods}

This is a retrospective study of all patients who were diagnosed with eclampsia at the University Hospital of UMDNJ between July 1998 and June 2008. Patients were identified based upon a discharge diagnosis of eclampsia. Medical records were then reviewed to confirm the diagnosis. Cases received

June 21, 2012

accepted

June 25, 2012

published online

January 25, 2013
Copyright (c) 2013 by Thieme Medical Publishers, Inc., 333 Seventh Avenue, New York, NY 10001, USA. Tel: +1(212) 584-4662.
DOI http://dx.doi.org/ 10.1055/s-0032-1329127. ISSN 2157-6998. 
Table 1 Highest blood pressure values antepartum, labor, postpartum, and at time of onset of seizure activity

\begin{tabular}{|l|l|l|l|l|}
\hline Case & Prenatal & Labor & Postpartum & Seizure activity \\
\hline 1 & $112 / 62$ & $142 / 78$ & $149 / 75$ (PPD 2); NSVD & $160 / 90$ (PPD 3) \\
\hline 2 & No PNC & $132 / 79$ & $155 / 92$ (recovery); repeat CD & $190 / 115$ (PPD 15) \\
\hline 3 & Outside PNC & Outside birth & Outside birth; repeat CD & $\begin{array}{l}134 / 91 \text { (PPD 11); seized at home-witnessed, } \\
\text { BP is upon arrival to ED }\end{array}$ \\
\hline 4 & $130 / 67$ & $142 / 85$ & $144 / 90$ (PPD 2); NSVD & $160 / 100$ (PPD 7) \\
\hline 5 & $129 / 67$ & $151 / 61$ & $131 / 60$ (PPD 2); NSVD & $130 / 80$ (PPD 5) \\
\hline
\end{tabular}

PNC, prenatal care; PPD, postpartum day; NSVD, normal spontaneous vaginal delivery; CD, cesarean delivery; BP, blood pressure; ED, emergency department.

confirmed to have LPE were then reviewed in detail. Information abstracted from the patient's medical record included medical history and physical, review of systems, and laboratory results prior to and after delivery. A descriptive analysis was then performed for the five confirmed cases with LPE. This research was approved by the institutional review board of the UMDNJ.

\section{Results}

Nineteen patients were confirmed to have eclampsia. Of these, seven seized postpartum with five of the seven seizing more than 48 hours after delivery (range 3 to 15 days postpartum). None of the five showed evidence of preeclampsia intrapartum or immediately postpartum and none received magnesium sulfate in labor. One of the patients did not have prenatal care. Of the four patients who had prenatal care (3 to 11 office visits), none showed evidence of preeclampsia before admission for delivery. Three of the five cases were hypertensive during their labor course, but none met criteria (e.g., proteinuria) for preeclampsia at the time. The range of blood pressure values in these three cases during labor ranged from 132 to $151 \mathrm{~mm}$ Hg systolic and 61 to $85 \mathrm{~mm} \mathrm{Hg}$ diastolic (see - Table 1).

At the time of seizure, three of the five patients had blood pressures in the severe range (see -Table 1). One of the patients who was normotensive upon arrival to the ED had a witnessed seizure at home where a blood pressure was not available. Two patients had proteinuria on the day of seizure activity, though one patient only developed proteinuria 5 days postseizure. Headache and visual disturbances were reported in two cases. Abnormal liver function test values were found in three of the five cases (aspartate aminotransferase 75 to $97 \mathrm{IU} / \mathrm{mL}$, alanine aminotransferase 62 to $82 \mathrm{IU} /$ $\mathrm{mL}$ ) and elevated uric acid values were found in two of the five patients ( 8.5 to $9.5 \mathrm{mg} / \mathrm{dL}$ ). All patients had normal serum creatinine and platelet counts. All patients had a negative neurodiagnostic test including head computed tomography or brain magnetic resonance imaging and/or a normal electroencephalogram.

\section{Discussion}

More than 25\% of eclamptic patients in this population met criteria for LPE. This is the same prevalence as another recent study published by Chames et $\mathrm{al}^{4}{ }^{4}$ representing a multicenter analysis of patients with eclampsia from March 1996 through February 2001. Data were collected regarding the relationship of the patient's first seizure to delivery. During the study period, 89 patients were diagnosed with eclampsia and 23 women (25\%) had LPE. Older studies including that of Leitch et $\mathrm{al}^{2}$ and Lubarsky et $\mathrm{al}^{3}$ reported a lower incidence of LPE, though Leitch indicated a rise in LPE over 60 years. These findings suggest that although intrapartum preeclampsia is commonly identified and treated, LPE has been poorly identified and prevented.

The authors of a recent Cochrane Review concluded that magnesium sulfate has been demonstrated to be effective as a seizure prophylaxis, as it more than halves the risk of eclampsia and probably reduces maternal death., ${ }^{5,6}$ The anticonvulsant activity may be mediated by magnesium's role as an N-methyl-D-aspartate (NMDA) antagonist, cerebral vasodilatation, and/or its calcium antagonist properties. As we continue to effectively administer prophylactic magnesium sulfate to peripartum preeclamptic patients, the proportion of patients with LPE (compared with the total incidence of eclampsia) may rise.

In our population, a total of $80 \%$ (95\% confidence interval [CI] 28 to $100 \%$ ) of LPE patients demonstrated hypertension and $40 \%$ (95\% CI 5 to $85 \%$ ), had headache at the time of onset of seizure activity. Although the $95 \% \mathrm{CI}$ indicates the wide range of true percent of cases with hypertension and headache prior to seizure, this is consistent with prodromal symptoms described in other reports of LPE. These findings suggest that patients with persistent hypertension following delivery may be at increased risk of LPE.

The limitations of this study include the small number of cases and retrospective design. Identification of patients was by medical records (International Classification of Diseases, Ninth Revision [ICD-9] coding) though with confirmation by direct medical record review. It is possible that patients with less typical presentations of eclampsia may have been excluded.

\section{Note}

This research was presented at the 2010 ACOG Annual Clinical Meeting in San Francisco, California. 


\section{References}

1 Chesley LC, Cosgrove RA. Remote deaths following eclampsia. Obstet Gynecol 1954;4:165-176

2 Leitch CR, Cameron AD, Walker JJ. The changing pattern of eclampsia over a 60-year period. $\mathrm{Br} \mathrm{J}$ Obstet Gynaecol 1997;104:917-922

3 Lubarsky SL, Barton JR, Friedman SA, Nasreddine S, Ramadan MK, Sibai BM. Late postpartum eclampsia revisited. Obstet Gynecol 1994;83:502-505
4 Chames MC, Livingston JC, Ivester TS, Barton JR, Sibai BM. Late postpartum eclampsia: a preventable disease? Am J Obstet Gynecol 2002;186:1174-1177

5 Duley L, Gülmezoglu AM, Henderson-Smart DJ, Chou D. Magnesium sulphate and other anticonvulsants for women with preeclampsia. Cochrane Database Syst Rev 2010;(11):CD000025

6 Duley L, Henderson-Smart D. Magnesium sulphate versus diazepam for eclampsia. Cochrane Database Syst Rev 2000;(2): CD000127 